\title{
Prior Vaccination and Effectiveness of Communication Strategies Used to Describe Infectious Diseases
}

\author{
Thomas S. Valley, Aaron M. Scherer, \\ Megan Knaus, Brian J. Zikmund-Fisher, \\ Enny Das, Angela Fagerlin
}

\begin{abstract}
Author affiliations: University of Michigan, Ann Arbor, Michigan, USA (T.S. Valley, A.M. Scherer, M. Knaus, B.J. Zikmund-Fisher); University of lowa, lowa City, lowa, USA (A.M. Scherer); Radboud University Nijmegen, Nijmegen, the Netherlands (E. Das); University of Utah School of Medicine, Salt Lake City, Utah, USA (A. Fagerlin); Informatics Decision-Enhancement and Analytic Sciences (IDEAS 2.0) Center for Innovation, Salt Lake City (A. Fagerlin)
\end{abstract}

DOI: https://doi.org/10.3201/eid2504.171408

We tested the effect of prior vaccination on response to communication strategies in a hypothetical news article about an influenza pandemic. Vaccinated were more likely than nonvaccinated participants to plan future vaccination, and future vaccination intent was greater with certain communication strategies. Using these findings to target communication may increase vaccination rates.

$\mathrm{V}$ accination rates for influenza remain surprisingly low (1). Despite goals to vaccinate $75 \%$ of high-risk Europeans by $2010,<50 \%$ had been vaccinated in 2013 (2). The reluctance of at-risk persons to receive vaccinations highlights the challenge of broadly vaccinating the general public.

Improving communication strategies that clinicians and healthcare organizations use to increase vaccination rates is cost-effective (3). Yet randomized trials to improve influenza vaccination rates by improving physicians' communication skills (4) or by using various public health messages (5) have not succeeded. Several studies have examined the effect of various communication strategies to improve vaccination rates for influenza (6-9). However, the greatest predictor of future vaccination is prior vaccination, and these studies assessed participants in aggregate (6). Guided by the Health Belief Model (10), we investigated whether experiences with prior vaccination might affect the effectiveness of certain communication strategies (Appendix, https://wwwnc.cdc.gov/EID/article/25/4/171408-App1.pdf).

Our study is a secondary analysis of a randomized experiment to test communication strategies and their effects on influenza immunization (6-9). After our study was deemed exempt from review by the University of Michigan Institutional Review Board, we recruited a stratified random sample of adults from a panel of Internet users through Survey Sampling International (https://www.survey sampling.com) (Appendix). We recruited participants from 11 countries: Finland $(n=1,554)$, Norway $(n=764)$, Sweden $(n=1,539)$, Hungary $(n=998)$, Poland $(n=1,509)$, Spain $(\mathrm{n}=1,604)$, Italy $(\mathrm{n}=1,509)$, Germany $(\mathrm{n}=1,546)$, the Netherlands $(\mathrm{n}=1,938)$, the United Kingdom $(\mathrm{n}=$ $1,762)$, and the United States $(\mathrm{n}=1,787)$.

Participants read a hypothetical news article that described the spread of influenza in their country. The article directly quoted hypothetical health experts and contained information about the influenza virus, its potential symptoms, and a vaccine in development. Articles were cross-randomized to provide participants with 5 varying communication strategies: 1) graphics (heat map, DOT map, picto-trendline) $(6)$; 2) case severity (severe, typical, both) (9); confident language (scientific certainty, uncertainty, uncertainty with normalizing language) (7); 4) influenza label (H11N3 influenza, horse flu, Yarraman flu) (8); and 5) metaphor use (infectious disease, war, gardening). The Appendix contains more information about communication strategies. Each news article contained all 5 communication strategies. The experiment used a $3 \times$ $3 \times 3 \times 3 \times 3$ between-subjects factorial design in which participants were randomly assigned to each communication strategy. After reading the newspaper article, participants were asked their vaccination status (whether they had received an influenza vaccination within the past 2 years) and intent to get vaccinated in the future (defined by a discrete visual analog scale ranging from 1 ["Definitely would not get a vaccination"] to 7 ["Definitely would get a vaccination"]).

We were interested in the main effect for an individual communication strategy depending on a participant's prior vaccination status. For each communication strategy, we conducted separate ordinal logistic regression models and included an interaction term of prior vaccination and the communication strategy of interest for each model. The dependent variable was intent to get vaccinated. As covariates, we included the participant's age, sex, and marital status and whether the participant was a healthcare worker. We estimated robust SEs with clustering by the participant's country of residence.

Of 20,138 participants, $16,401(81 \%)$ completed the survey; of these, 4,999 (30\%) had received an influenza vaccination within the previous 2 years and 11,402 $(70 \%)$ had not. The average age was $51.4(\mathrm{SD} \pm 16.9)$ for vaccinated and 44.9 (SD \pm 15.4 ) for nonvaccinated participants. Approximately $44.6 \%$ of vaccinated and $52.1 \%$ of nonvaccinated participants were female (Appendix Table 1). 
Table. Effect of communication strategies on intent for future influenza vaccination, by influenza vaccination status

\begin{tabular}{|c|c|c|c|c|c|}
\hline \multirow[b]{2}{*}{ Strategy } & \multicolumn{4}{|c|}{ Vaccination over previous $2 \mathrm{y}$, adjusted odds ratio $(95 \% \mathrm{Cl})^{*}$} & \multirow{2}{*}{$\begin{array}{c}p \text { value for } \\
\text { interactiont }\end{array}$} \\
\hline & No & $p$ value & Yes & $\mathrm{p}$ value & \\
\hline Graph type & & & & & $<0.001$ \\
\hline Picto-trendline & Referent & & Referent & & \\
\hline DOT map & $1.1(0.9-1.2)$ & 0.06 & $1.0(0.9-1.1)$ & 0.92 & \\
\hline Heat map & $1.1(1.0-1.2)$ & 0.01 & $1.1(0.9-1.2)$ & 0.08 & \\
\hline Case severity & & & & & $<0.001$ \\
\hline Both & Referent & & Referent & & \\
\hline Typical & $1.0(0.9-1.1)$ & 0.78 & $0.9(0.8-1.0)$ & 0.07 & \\
\hline Severe & $1.1(1.0-1.3)$ & 0.02 & $1.1(0.9-1.2)$ & 0.43 & \\
\hline Confident language & & & & & $<0.001$ \\
\hline Uncertainty with normalizing language & Referent & & Referent & & \\
\hline Uncertainty & $1.0(0.9-1.1)$ & 0.97 & $1.1(0.9-1.2)$ & 0.12 & \\
\hline Scientific certainty & $1.2(1.1-1.3)$ & $<0.001$ & $1.3(1.1-1.4)$ & $<0.001$ & \\
\hline Influenza label & & & & & $<0.001$ \\
\hline Horse & Referent & & Referent & & \\
\hline H11N3 & $1.0(0.9-1.1)$ & 0.62 & $1.4(1.1-1.7)$ & 0.001 & \\
\hline Yarraman & $1.1(1.0-1.2)$ & 0.001 & $1.2(1.1-1.4)$ & 0.001 & \\
\hline Metaphor use & & & & & $<0.001$ \\
\hline Infectious disease & Referent & & Referent & & \\
\hline War & $1.0(0.9-1.1)$ & 0.78 & $1.0(0.9-1.1)$ & 0.60 & \\
\hline Gardening & $1.0(0.9-1.1)$ & 0.75 & $1.0(0.9-1.1)$ & 0.41 & \\
\hline
\end{tabular}

Our results showed that previously vaccinated participants were more likely than nonvaccinated participants to plan for future vaccinations (adjusted odds ratio 5.8, $95 \%$ CI $4.8-7.0 ; \mathrm{p}<0.001)$. We found significant interaction effects between prior vaccination and each communication strategy ( $<<0.001$ for each strategy) (Table; Appendix Table 2). However, this effect varied according to the type of communication strategy. Nonvaccinated participants reported greater intent for future vaccination when heat maps, severe cases, confident language, or exotic influenza labels were used (Table). Vaccinated participants reported greater intent for future vaccination when confident language or scientific/exotic influenza labels were used (Table). The use of metaphors had no effect on either group.

This study should be interpreted in the context of certain limitations. For instance, participants reviewed a hypothetical news article, which may be different than direct communication with a healthcare provider or reading an actual article during a pandemic.

Certain communication strategies, such as use of confident language or an exotic influenza label, were effective regardless of prior vaccination status. Yet use of a scientific influenza label was more effective than use of an exotic influenza label among previously vaccinated participants. Other communication strategies, such as use of heat maps or describing severe cases, were effective among nonvaccinated but not previously vaccinated participants. Vaccination rates for influenza may be improved by targeting healthcare communication based on prior vaccination experiences $(11,12)$.

\section{Acknowledgments}

This work was supported by the European Union's Seventh Framework Programme for research, technological development and demonstration under grant agreement \#278763 (to A.F.) and the National Institutes of Health, K23HL140165 (to T.S.V.). Funding sources had no role in the study conception, design, conduct, analysis, or manuscript construction.

\section{About the Author}

Dr. Valley is an assistant professor in the Division of Pulmonary and Critical Care Medicine at the University of Michigan. His research focuses on improving clinical decision making in pulmonary disease and critical care.

\section{References}

1. European Centres for Disease Prevention and Control. Seasonal influenza vaccination in Europe-overview of vaccination recommendations and coverage rates in the EU Member States for the 2012-13 influenza season [cited 2017 Aug 2]. http://dx.doi.org/10.2900/693898

2. World Health Organization. Influenza (seasonal) fact sheet. Geneva: The Organization; 2014.

3. Goldstein S, MacDonald NE, Guirguis S; SAGE Working Group on Vaccine Hesitancy. Health communication and vaccine hesitancy. Vaccine. 2015;33:4212-4. http://dx.doi.org/10.1016/ j.vaccine.2015.04.042

4. Henrikson NB, Opel DJ, Grothaus L, Nelson J, Scrol A, Dunn J, et al. Physician communication training and parental vaccine hesitancy: a randomized trial. Pediatrics. 2015;136:70-9. http://dx.doi.org/10.1542/peds.2014-3199

5. Frew PM, Kriss JL, Chamberlain AT, Malik F, Chung Y, Cortés $\mathrm{M}$, et al. A randomized trial of maternal influenza immunization decision-making: a test of persuasive messaging 
models. Hum Vaccin Immunother. 2016;12:1989-96. http://dx.doi.org/10.1080/21645515.2016.1199309

6. Fagerlin A, Valley TS, Scherer AM, Knaus M, Das E, Zikmund-Fisher BJ. Communicating infectious disease prevalence through graphics: results from an international survey. Vaccine. 2017;35:4041-7. http://dx.doi.org/10.1016/j.vaccine.2017.05.048

7. Han PKJ, Zikmund-Fisher BJ, Duarte CW, Knaus M, Black A, Scherer AM, et al. Communication of scientific uncertainty about a novel pandemic health threat: ambiguity aversion and its mechanisms. J Health Commun. 2018;23:435-44. http://dx.doi.org/ 10.1080/10810730.2018.1461961

8. Scherer AM, Knaus M, Zikmund-Fisher BJ, Das E, Fagerlin A. Effects of influenza strain label on worry and behavioral intentions. Emerg Infect Dis. 2017;23:1425-6. http://dx.doi.org/10.3201/eid2308.170364

9. Zikmund-Fisher BJ, Scherer AM, Knaus M, Das E, Fagerlin A. Discussion of average versus extreme case severity in pandemic risk communications. Emerg Infect Dis. 2017;23:706-8. http://dx.doi.org/10.3201/eid2304.161600

10. Champion V, Skinner C. The health belief model. In: Glanz K, Rimer BK, Viswanath K, editors. Health behavior and health education: theory, research, and practice, 4 th ed. San Francisco: Jossey-Bass; 2008. p. 45-67.

11. Noar SM, Benac CN, Harris MS. Does tailoring matter? Metaanalytic review of tailored print health behavior change interventions. Psychol Bull. 2007;133:673-93. http://dx.doi.org/ 10.1037/0033-2909.133.4.673

12. Cappella JN. Integrating message effects and behavior change theories: organizing comments and unanswered questions. Journal of Communication. 2006;56(suppl1):S265-79.

Address for correspondence: Thomas S. Valley, University of Michigan, Division of Pulmonary and Critical Care Medicine, 2800 Plymouth Rd, Bldg 16-G028W, Ann Arbor, MI 48109, USA; email: valleyt@umich.edu

\section{Peripheral Plasma and Semen Cytokine Response to Zika Virus in Humans}

\section{Jean-Michel Mansuy, ${ }^{1}$ Hicham El Costa, ${ }^{1}$ Jordi Gouilly, Catherine Mengelle, Christophe Pasquier, Guillaume Martin-Blondel, Jacques Izopet, Nabila Jabrane-Ferrat}

Author affiliations: Centre Hospitalier Universitaire de Toulouse, Toulouse, France (J.-M. Mansuy, H. El Costa, C. Mengelle, C. Pasquier, G. Martin-Blondel, J. Izopet); INSERM U1043-CNRS UMR5282 Université Toulouse III, Toulouse (H. El Costa,

J. Gouilly, G. Martin-Blondel, J. Izopet, N. Jabrane-Ferrat)

DOI: https://doi.org/10.3201/eid2504.171886

${ }^{1}$ These authors contributed equally to this article.
We assessed Zika virus RNA and select cytokine levels in semen, blood, and plasma samples from an infected patient in South America. Viral RNA was detected in semen $>2$ months after viremia clearance; cytokine profiles differed in semen and plasma. After viremia, Zika virus appears to become compartmentalized in the male reproductive tract.

$\mathrm{B}$ efore the 2015-2016 outbreak, Zika virus infection had been associated with only mild symptoms. However, the outbreak revealed infection could cause severe clinical manifestations, particularly for fetuses and newborns (1). Furthermore, detection of replicative virus in semen and sexual transmission of the infection resulted in a paradigm shift in Zika virus virology $(2,3)$. Several animal models were developed to study these phenomena, and studies revealed that Zika virus persistence within the male reproductive tract (MRT) results in diminished testosterone and oligospermia (4). However, because of complex ethics considerations, the consequences of infection on the MRT remain poorly understood (5).

To characterize infection in the MRT further, we conducted a longitudinal 6-month study examining Zika virus load and immunologic profile in blood, plasma, and semen in 1 man. The study patient was a 32-year-old immunocompetent white man with an asymptomatic Zika virus infection acquired in South America in January 2016; the control was a healthy 40 -year-old white man without risk factors for acute or chronic infection who lived in the same area. We evaluated the concentrations of a select panel of cytokines, including innate immune mediators (interferon [IFN] $-\gamma$, interleukin [IL]-15, IFN- $\beta$ ); inflammatory factors (IL-6, IL-18, soluble intercellular adhesion molecule 1 [sICAM-1]); chemokines (CC-motif chemokine ligand [CCL] 3, CCL-4, CXC-motif chemokine ligand [CXCL] 1, CXCL-8, CXCL-10); hematopoietic factors (granulocyte colony-stimulating factor [G-CSF], granulocyte-macrophage colony-stimulating factor); the angiogenic factor vascular endothelial growth factor A (VEGF-A); and proteases (matrix metalloproteinase [MMP]-2, MMP-9). We quantified cytokines using ProcartaPlex Multiplex Assay (ebioscience, https://www.thermofisher.com).

At admission, the patient had moderate fever, maculopapular rash, myalgia, and arthralgia and recovered within a few days. He was HIV negative; dengue and chikungunya virus infections were ruled out using ELISA Diapro (Diagnostic Bioprobes Srl, https://www.diapro.it) and RealStar Dengue and Chikungunya RT-PCR Kit 2.0 (Altona Diagnostics, https://www.altona-diagnostics.com). The patient did not experience other genital or urinary tract infections during the study.

Two days after symptom onset, viral RNA was higher in semen $\left(1.04 \times 10^{5}\right.$ copies $\left./ \mathrm{mL}\right)$ than in blood $\left(9.4 \times 10^{3}\right.$ copies $/ \mathrm{mL}$ ); RNA was detectable for up to 100 days in blood 